J. Clin. Chem. Clin. Biochem.

Vol. 14, 1976, pp. 197-202

\title{
Lipoprotein-X bei Neugeborenen: Gehäuftes Auftreten ohne nachweisbare Cholestase
}

\author{
Von Irene Witt und Marieluise Ober
}

Biochemisches Labor der Universitäts-Kinderklinik Freiburg i. Br.

(Eingegangen am 1. September/17: Dezember 1975)

\section{Herrn Professor Dr. J. Kühnau zum 75. Geburtstag gewidmet}

Zusammenfassung: Die Untersuchung von 194 Neugeborenen zeigte, daß bei etwa 50\% der Kinder in den ersten Lebenswochen das abnormale Lipoprotein-X (LP-X) im Serum nachgewiesen werden kann, ohne daß klinischchemisch ein Hinweis für eine Cholestase besteht. Bei Früh- und Mangelgeborenen war der Anteil der LP-X-positiven Kinder noch höher (65\%).

Der Nachweis von LP-X war unabhängig von der Höhe der Gesamt-Bilirubin-Konzentration. Die Aktivitäten der Leucin-Arylamidase (EC 3.4.1.1) und der $\gamma$-Glutamyltransferase (EC 2.3.2.2) sowie die Konzentrationen des gesamten und freien Cholesterins zeigten keine Unterschiede zwischen den LP-X-positiven und LP-X-negativen Kindern.

Von einer Ausnahme abgesehen, trat LP-X nie am ersten Lebenstag, frühestens am zweiten Lebenstag auf. Bei einigen Kindern, die kurz nach der Geburt LP-X-positiv waren, konnte LP-X auch noch im Alter von 2-3 Monaten nachgewiesen werden.

Kinder mit Blutgruppen-Inkompatibilität waren nicht häufiger LP-X-positiv als Kinder mit unspezifischer Hyperbilirubinämie. Austauschtransfusionen führten häufig zum Verschwinden von LP-X im Serum. Später konnte es jedoch dann wieder nachgewiesen werden.

Bei einigen Kindern, die in den ersten Lebenstagen LP-X-negativ waren, trat LP-X erst im Alter von 2-3 Monaten im Serum auf. Der LP-X-Test kann im Neugeborenenalter nicht zur Diagnostik einer Cholestase eingesetzt werden. Erst jenseits des 1. Lebensjahres ist er Cholestase-spezifisch.

Das gehäufte Auftreten von LP-X bei Neugeborenen wird als „Unreife“ der Leberfunktion diskutiert.

\section{LP-X in newborns: increased incidence of positive tests without cholestasis}

Summary: The investigation of 194 newborns has shown that during the first weeks of life the abnormal lipoprotein$X(L P-X)$ was present in the serum of nearly $50 \%$ of the infants, with no clinical chemical evidence of cholestasis. The percentage of LP.X positive tests was even higher in the group of immature newborns (65\%). There was no correlation between the bilirubin concentration and the detection of LP-X. The activities of leucine arylamidase (EC 3.4.1.1) and $\gamma$-glutamyltransferase (EC 2.3.2.2) as well as the concentrations of total and free cholesterol did not differ in the LP-X positive and negative infants.

Except in one case; LP-X was never detectable on the first day of life. The earliest date of appearance was the second day. In the serum of some infants, who were LP-X positive shortly after birth, the lipoprotein could still be found at the age of $2-3$ months.

The incidence of LP.X was not higher in newborns with blood group incompatibility than in newborns with unspecific hyperbilinubinaemia. After exchange transfusions LP-X disappeared in most cases, but it could later often be detected again. In some newborns, who were LP-X negative a feww days after birth LP-X was first detected at the age of 2-3 months.

The LP-X test is of no use for the diagnosị of cholestasis in newborn infants. The test is specific for cholestasis only after the first year of life.

The increased incidence of positive LP-X tests in newborns is discussed as a consequence of immature liver function. 


\section{Einführung}

Nach den Arbeiten von Seidel et al. $(1,2,3)$ sowie von Ritland et al. (4) ist das Auftreten des abnormalen lowdensity Lipoproteins (LP-X) im Serum eines Patienten ein signifikanter Hinweis für eine Cholestase.

Die Einführung der LP-X-Bestimmung schien auch für eine pädiatrische Klinik nützlich zu sein, z. B. zum Nachweis einer angeborenen Gallengangs-Atresie bzw. -Hypoplasie oder zum Nachweis einer Cholestase bei Hepatitis, zumal Seidel et al. (3) bei Neugeborenen mit hämolytischem Ikterus in keinem der untersuchten Fälle $(n=58)$ LP-X im Serum nachgewiesen hatten.

Zur Einführung der Methode wurde die LP-X-Bestimmung u. a. auch bei Neugeborenen durchgefüht, ohne daß eine Indikation dafür vorlag. Dabei wurden zahlreiche positive LP-X-Tests bei Kindern gefunden, ohne daß klinisch-chemisch eine Cholestase nachweisbar war. Der Test mußte daher für Neugeborene bald als unspezifisch angesehen werden (5). $\mathrm{Zu}$ dem gleichen Ergebnis kamen auch Lachmann et al. (6).

Da das gehäufte Auftreten von LP-X bei Neugeborenen aus biochemischer Sicht sehr interessant ist, wurde eine eingehende Studie über das Auftreten von LP-X bei Neugeborenen und Frühgeborenen durchgeführt, über deren erste Ergebnisse berichtet werden soll.

Neben dem zeitlichen Auftreten von LP-X nach der Geburt wurde die Abhängigkeit von Blutgruppen-Inkompatibilitäten, von Austauschtransfusionen und von der Bilirubin-Konzentration untersucht. Zur Klärung der Frage, ob das Vorhandensein von LP-X bei Neugeborenen mit einer "physiologischen Unreife“ der Ausscheidungsfunktion der Leber verbunden ist, wurden die Aktivitäten der $\gamma$-Glutamyltransferase (EC 2.3.2.2) und LeucinArylamidase (EC 3.4.1.1), sowie die Konzentrationen des gesamten und freien Cholesterins bestimmt.

\section{Methoden}

Alle Bestimmungen wurden in Serum nach venöser Blutentnahme durchgeführt.

Der LP-X-Nachweis erfolgte nach Trennung durch AgargelElektrophorese mittels Polyanionenpräzipitation (Rapidophor, Immuno AG, Heidelberg).

Die Aktivitätsbestimmung der Leucin-Arylamidase und der $\gamma$-Glutamyltransferase erfolgte als Mikromethode (Enzymautomat Eppendorf) mit Testkombinationen der Firma Boehringer Mannheim, GmbH.

Bilirubin wurde mit der Methode von Jendrassik \& Grof (7) als Halbmikrobestimmung gemessen. Cholesterin (gesamt und frei) wurde enzymatisch als Mikromethode mit der Testkom- bination der Firma Boehringer Mannheim, GmbH, bestimmt.

\section{Ergebnisse}

Der Lipoprotein-X-Nachweis wurde im Serum von 194 Kindern durchgeführt. Die untersuchten Kinder verteilten sich auf folgende drei Gruppen:
I. „Ausgewählte“ Neugeborene mit Hyperbilirubinämie,

II. „Nichtausgewählte“ reife Neugeborene mit Hyperbilirubinämie,

III. „Nichtausgewählte“ Frühgeborene und Mangelgeborene.

\section{Gruppe I}

Es wurden insgesamt 56 Neugeborene mit Hyperbilirubinämie untersucht, deren Geburtsgewichte sowohl geringer als auch höher als $2500 \mathrm{~g}$ waren. Die Kinder stellen insofern eine „ausgewählte“ Gruppe dar, als die Befundanalyse retrospektiv durchgeführt wurde und dabei vermutlich LP-X-positive Neugeborene vermehrt registriert wurden.

In dieser Gruppe wurde bei 47 Kindern LP-X im Serum nachgewiesen. Bei 9 der LP-X-positiven Kinder sprachen klinische Symptome und Laborbefunde für eine Lebererkrankung, die vermutlich mit einer Cholestase einherging. Bei den übrigen 38 LP-X-positiven Kindern ( $=68 \%$ ) war keine Cholestase nachweisbar.

Früh- und Mangelgeborene dieser Gruppe waren nicht häufiger LP-X-positiv als reife Neugeborene. Auch war kein gehäuftes Auftreten von LP-X bei Kindern mit einer Blutgruppen-Inkompatibilität nạchweisbar (vgl. Tab. 1).

\section{Gruppe II}

In dieser ,nichtausgewählten“ Gruppe wurden insgesamt 88 reife Neugeborene untersucht, die in der Mehrzahl eine Hyperbilirubinämie hatten. Die Geburtsgewichte lagen über $2500 \mathrm{~g}$.

Bei 43 dieser Kinder wurde während der ersten 4 Lebenswochen einmal oder mehrmals LP-X im Serum nachgewiesen. Bei 3 der LP-X-positiven Kinder sprachen klinische Symptome und Laborbefunde für eine Lebererkrankung, die vermutlich mit einer Cholestase einherging. Bei den übrigen 40 Kindern ( $=46 \%$ ), die LP-Xpositiv waren, konnte keine Cholestase nachgewiesen werden.

Bei Kindern mit einer Blutgruppen-Inkompatibilität trat LP-X nicht gehäuft auf (vgl. Tab. 1).

\section{Grup p pe III}

In dieser „nichtausgewählten “ Gruppe wurden insgesamt 50 Früh- oder Mangelgeborene untersucht. Die Geburtsgewichte lagen unter $2500 \mathrm{~g}$. Bei 32 dieser Kinder $(=65 \%)$ wurde während der ersten 6 Lebenswochen einmal oder mehrmals LP.X im Serum nachgewiesen. Bei keinem der LP-X-positiven Kinder sprachen klinische Symptome oder Laborbefunde für eine Chole- 
Tab. 1. Zusammenstellung der 194 untersuchten Neugeborenen.

\begin{tabular}{|c|c|c|c|c|c|c|c|c|}
\hline \multirow[t]{2}{*}{ Gruppe } & \multirow[t]{2}{*}{ Anzahl } & \multicolumn{4}{|c|}{ LP-X-positive Neugeborene } & \multicolumn{3}{|c|}{ LP-X-negative Neugeborene } \\
\hline & & $\begin{array}{l}\text { Verdacht } \\
\text { auf } \\
\text { Cholestase }\end{array}$ & $\begin{array}{l}\text { Reife } \\
>2500 \mathrm{~g}\end{array}$ & $\begin{array}{l}\text { Früh- u. } \\
\text { Mangel- } \\
\text { geborene } \\
>2500 \mathrm{~g}\end{array}$ & $\begin{array}{l}\text { Blut- } \\
\text { gruppen } \\
\text { Inkompa- } \\
\text { tibilität }\end{array}$ & $\begin{array}{l}\text { Reife } \\
>2500 \mathrm{~g}\end{array}$ & $\begin{array}{l}\text { Früh- u. } \\
\text { Mangel- } \\
\text { geborene } \\
>2500 \mathrm{~g}\end{array}$ & $\begin{array}{l}\text { Blut- } \\
\text { gruppen } \\
\text { Inkompa- } \\
\text { tibilität }\end{array}$ \\
\hline \multicolumn{9}{|l|}{1} \\
\hline $\begin{array}{l}\text { "Ausgewählte“" } \\
\text { Neugeborene mit } \\
\text { Hyperbilirubinämie }\end{array}$ & 56 & 9 & 30 & 17 & 6 & 5 & 4 & 1 \\
\hline \multicolumn{9}{|l|}{ II } \\
\hline $\begin{array}{l}\text { "Nichtausgewählte“ } \\
\text { reife Neugeb. mit } \\
\text { Hyperbilirubinämie }\end{array}$ & 88 & 3 & 43 & & 9 & 45 & & 6 \\
\hline \multicolumn{9}{|l|}{ III } \\
\hline $\begin{array}{l}\text { „Nichtausgewählte“ } \\
\text { Frühgeborene u. } \\
\text { Mangelgeborene }\end{array}$ & 50 & 0 & & 32 & 2 & & 17 & 1 \\
\hline
\end{tabular}

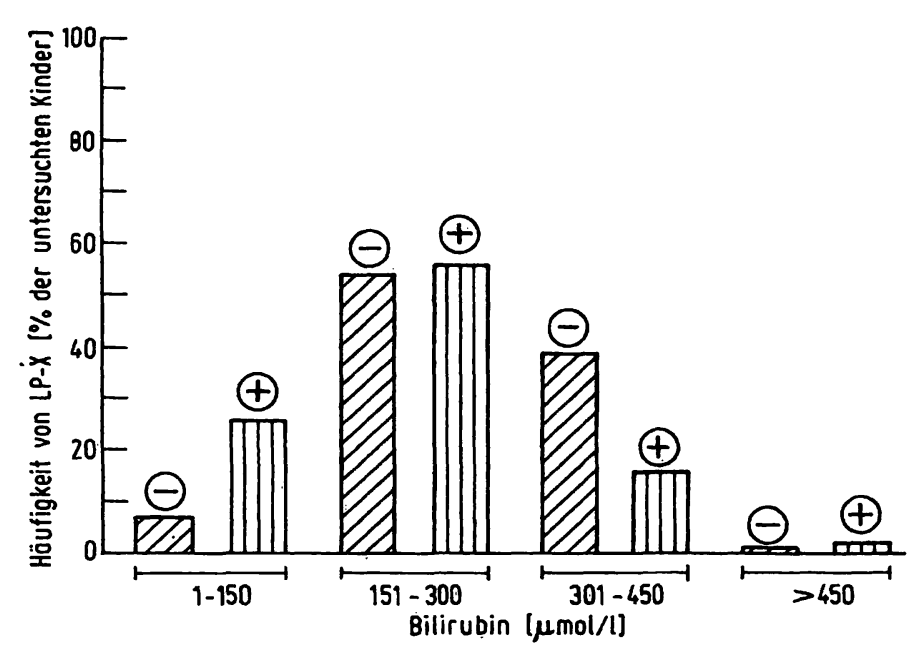

Abb. 1. Prozentuale Verteilung der insgesamt untersuchten LP-Xpositiven und -negativen $K$ inder nach den verschiedenen Bilirubin-Konzentrationen.

stase. Bei Kindern mit einer Blutgruppen-Inkompatibilität war LP-X nicht gehäuft nachweisbar (vgl. Tab. 1).

In Abbildung 1 ist das Auftreten des abnormalen Lipoproteins zur Höhe der Gesamt-Bilirubin-Konżentration in Beziehung gesetzt. Daraus geht hervor, daß die LP-Xpositiven Kinder eher niedrigere, keineswegs aber höhere, Bilinubin-Konzentrationen hatten als die LP-X-negativen Kinder. Es bestèht also keine Korrelation zwischen der Höhe der Gesamt-Bilirubin-Konzentration und dem Auftreten von LP-X.

Die sogenannten „Cholestase-anżeigenden“ Enżyme Leucin:Arylamidase und $\gamma$-Glutamyltransferase zeigten im Mittel bei den LP-X-positiven Kindern keine erhöhten Aktivitäten (vgl. Tab. 2). In dieser Zusammen= stellung sind die Werte der Kinder, bei denen der Ver-
Tab. 2. Aktivitäten der Leucin-Arylamidase (EC 3.4.1.1) und der $\gamma$-Glutamyltransferase (EC 2.3.2.2) im Serum von LP-Xpositiven und LP-X-negativen Neugeborenen $(1 .-30$. Lebenstag).

\begin{tabular}{lllll}
\hline & $\begin{array}{l}\text { Leucin- } \\
\text { Arylamidase }\end{array}$ & $\begin{array}{l}\gamma \text {-Glutamyl- } \\
\text { transferase }\end{array}$ \\
\hline & $\frac{\mathrm{U} / 1}{\mathrm{x}}$ & $\pm \mathrm{s}$ & $\frac{\mathrm{U} / \mathrm{l}}{\mathrm{x}}$ & $\pm \mathrm{s}$ \\
& 19 & 7 & 65 & 29 \\
$\begin{array}{l}\text { LP-X-positive Neugeborene } \\
(\mathrm{n}=32)\end{array}$ & 22 & 11 & 52 & 24 \\
$\begin{array}{l}\text { LP-X-negative Neugeborene } \\
(\mathrm{n}=34)\end{array}$ & bis 22 & bis 100 \\
\begin{tabular}{l} 
Normbereich \\
\hline
\end{tabular} & &
\end{tabular}

dacht auf eine Cholestase bestand (vgl. Tab. 1), nicht einbezogen. Die Aktivität der alkalischen Phosphatase wurde nicht zum Vergleich mit herangezogen, da sie im Kindesalter zu stark von Knochenprozessen beeinflußt wird.

Da bei Patienten mit einem angeborenen Mangel an Lecithin-Cholesterol-Acyltransferase LP-X im Serum gefunden wird $(8,9)$, stand zur Diskussion, ob bei Neugeborenen eine verminderte Aktivität des Enzyms vorliegen könnte. Da die Lecithin-Cholesterol-Acyltransferase-Aktivität nicht direkt gemessen werden konnte, versuchten wir einen Lecithin-Cholesterol-Acyltransferase-Mangel indirekt über die Bestimmung des gesamten und freien Cholesterins auszzuschließen. Patienten mit Lecithin-Cholestrol-Acyltransferase-Mangel haben hohe Konzentrationen an freiem Cholesterin (etwa 80-90\% des Gesamt-Cholesterins) und entsprechend niedrige Konzentrationen an verestertem Cholesterin (9). Tabelle 3 zeigt, daß Neugeborene keine erhöhten Konzentrationen an freiem 
Tab. 3. Serum-Cholesterin-Konzentrationen von LP-X-positiven und LP-X-negativen Neugeborenen.

\begin{tabular}{|c|c|c|c|c|}
\hline & \multicolumn{2}{|c|}{$\begin{array}{l}\text { Gesamt- } \\
\text { Cholesterin }\end{array}$} & $\begin{array}{l}\begin{array}{l}\text { Freies } \\
\text { Cholesterin }\end{array} \\
\mathrm{mmol} / \mathrm{l}\end{array}$ & $\begin{array}{l}\text { Verestertes } \\
\text { Cholesterin }\end{array}$ \\
\hline & $\overline{\mathbf{x}}$ & $\pm s$ & $\pm s$ & $\pm s$ \\
\hline $\begin{array}{l}\text { LP-X-positive } \\
\text { Neugeborene } \\
(n=55)\end{array}$ & 3,24 & 0,75 & $0,520,18$ & $2,720,73$ \\
\hline $\begin{array}{l}\text { LP-X-negative } \\
\text { Neugeborene } \\
(n=45)\end{array}$ & 3,21 & 0,73 & $0,52 \quad 0,21$ & $2,69 \quad 0,70$ \\
\hline
\end{tabular}

Cholesterin haben. Außerdem sind die CholesterinKonzentrationen der LP-X-positiven Kinder gegenüber denen der LP-X-negativen Kinder nicht verändert. Verminderte Lecithin-Cholesterol-Acyltransferase-Aktivität der Neugeborenen ist daher äußerșt unwahrscheinlich.

Um die Frage zu klären, ob LP-X eventuell ein nur in der Fetalzeit vorliegendes Lipoprotein ist und in bestimmten Fällen noch nach der Geburt vorkommt, untersuchten wir 100 Frühgeborene am 1. Lebenstag auf das Vorliegen von LP-X. Nur bei einem der $100 \mathrm{Kin}$ der wurde LP-X am 1. Lebenstag gefunden. Es handelte sich dabei um ein unreifes Frühgeborenes mit einem Hydrops bei Erythroblastose, das noch am 1. Lebenstag verstarb.

Über das zeitliche Auftreten von LP-X im Serum der Neugeborenen haben wir darüber hinaus folgende

Tab. 4. Beispiele für das zeitliche Auftreten von LP-X bei reifen Neugeborenen und Frühgeborenen mit Hyperbilirubinämie ohne Austauschtransfusionen.

$+=$ positiv, $(+)=$ schwach positiv, $\phi=$ negativ, LT $=$ Lebenstag.

\begin{tabular}{|c|c|c|c|c|c|}
\hline $\begin{array}{l}\text { Reifes } \\
\text { Neugeborenes } \\
3680 \mathrm{~g}\end{array}$ & 1. LT & $\begin{array}{l}\text { 3. LT } \\
(+)\end{array}$ & $\begin{array}{l}\text { 4. } \hat{\mathrm{LT}} \\
(+)\end{array}$ & $\begin{array}{l}\text { 5. LT } \\
\phi\end{array}$ & \\
\hline $\begin{array}{l}\text { Früh- } \\
\text { geborenes } \\
2500 \mathrm{~g}\end{array}$ & $\begin{array}{l}\text { 6. LT } \\
+\end{array}$ & $\underset{\phi}{12 .} \mathrm{LT}$ & & & \\
\hline $\begin{array}{l}\text { Früh- } \\
\text { geborenes } \\
1970 \mathrm{~g}\end{array}$ & $\begin{array}{l}\text { 1. LT } \\
\phi\end{array}$ & $\begin{array}{l}\text { 3. LT } \\
(+)\end{array}$ & $\begin{array}{l}\text { 4. LT } \\
(+)\end{array}$ & $\begin{array}{l}\text { 20. LT } \\
+\end{array}$ & $\begin{array}{l}1 \text { Monat } \\
\phi\end{array}$ \\
\hline $\begin{array}{l}\text { Früh- } \\
\text { geborenes } \\
2400 \mathrm{~g}\end{array}$ & ${ }_{\phi}^{1 .} \mathrm{LT}$ & $\begin{array}{l}\text { 3. LT } \\
+\end{array}$ & $\begin{array}{l}\text { 8. LT } \\
+\end{array}$ & $\begin{array}{l}\text { 13. LT } \\
(+)\end{array}$ & - \\
\hline $\begin{array}{l}\text { Früh- } \\
\text { geborenes } \\
1530 \mathrm{~g}\end{array}$ & $\begin{array}{l}\text { 9. LT } \\
+\end{array}$ & $\begin{array}{l}\text { 10. LT } \\
+\end{array}$ & $\begin{array}{l}1 \text { Monat } \\
+\end{array}$ & $\cdot$ & \\
\hline $\begin{array}{l}\text { Früh- } \\
\text { geborenes } \\
2500 \mathrm{~g}\end{array}$ & $\begin{array}{l}\text { 5. LT } \\
+\end{array}$ & $\begin{array}{l}\text { 6. LT } \\
+\end{array}$ & $\begin{array}{l}2 \text { Monate } \\
+\end{array}$ & & \\
\hline
\end{tabular}

Beobachtungen gemacht: Bei einigen Kindern trat LP-X in den ersten Lebenstagen auf und war dann nach einigen Tagen nicht mehr nachweisbar. Vielfach blieb der Nachweis aber mehrere Monate positiv. Da die Kinder nur zur Durchführung des LP-X-Tests nicht wieder in die Klinik einbestellt werden konnten, können wir über das Verschwinden von LP-X in diesen Fällen keine Aussage machen (vgl. Täb. 4).

Bei LP-X-positiven Kindern war der LP-X-Nachweis nach einer Austauschtransfusion in der Regel negativ. Häufig trat das Lipoprotein dann abèr emeut auf (vgl. Tab. 5).

Bei der ambulanten Nachkontrolle von AustạschKindern fiel auf, daß Kinder, die in den ersten Lebenstagen LP-X-negativ waren, im Alter von 1-3 Monaten LP:X-positiv waren.

Der genaue Zeitpunkt des Auftretens von LP-X kann dabei nicht angegeben werden. Die Vermutung, daß Kinder nach Austauschtransfusionen gehäuft LP-X-positiv sind, nuß in einer weiteren Untersuchung überprüft werden.

Tab. 5. Beispiele für das zeitliche Auftreten von LP-X bei reifen Neugeborenen und Frühgeborenen mit Austauschtransfusionen.

$+=$ positiv,$(+)=$ schwach positiv, $\phi=$ negativ, LT = Lebenstag, $A T=$ Austausch, $\mathrm{M}=$ Monat.

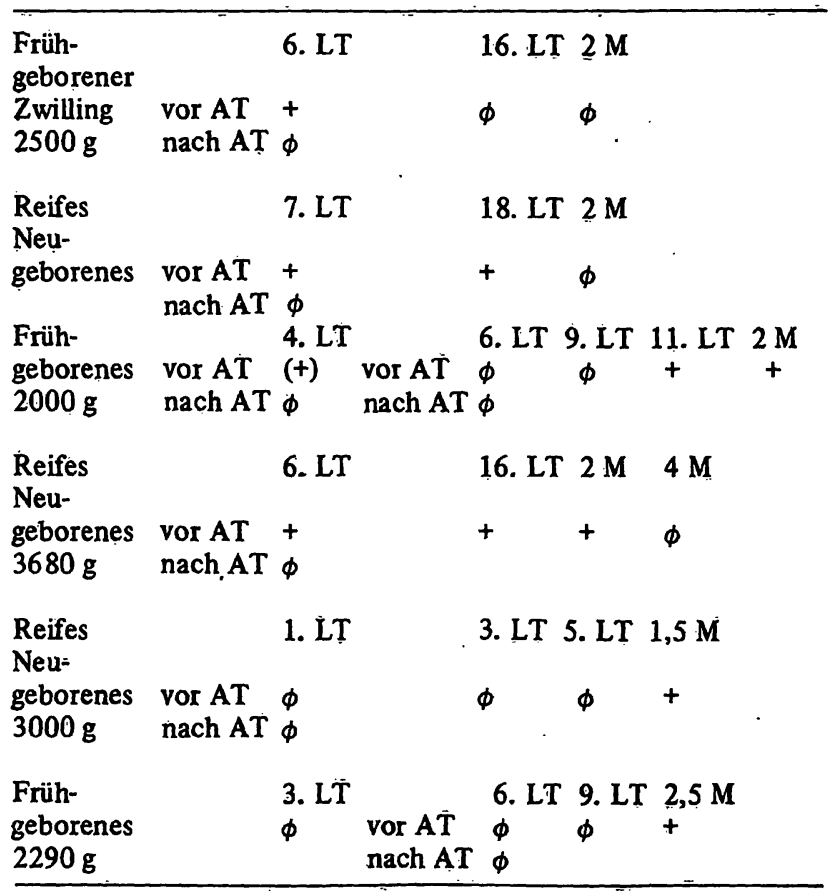

\section{Diskussion}

Die vorliegenden Untersuchungen haben gezeigt, daß der - LP-X-Test im Neugeborenenalter nicht zum Nachweis eịler Cholestase eingesetżt werden kann, da mindestens $50 \%$ aller Neugeborenen in den ersten Lebenswochen für kürzere oder längere Żeit LP-X-positiv sind. 
Aus unseren Beobachtungen über das zeitliche Auftreten von LP-X muß man schließen, daß der Prozentsatz der LP-X-positiven Neugeborenen noch höher liegt. Da das Auftreten des Lipoproteins nach der Geburt ganz unregelmäßig ist und in den meisten Fällen der LP-X-Test nur im Zusammenhang mit der Blutabnahme für andere Untersuchungen erfolgte, war der positive Nachweis häufig rein zufällig.

Die regelmäßige Abnahme an bestimmten Lebenstagen hätte diese Zufälligkeit nur bei sehr kurz aufeinander folgenden Blutabnahmen verhindert, was bei sonst gesunden Kindern nicht durchfuhrbar ist.

Bei der Untersuchung des Serums von 30 gesunden Kindern im Alter von 8-14 Monaten wurde LP-X in keinem Fall gefunden, so daß bei über $1 \mathrm{Jahr}$ alten Kindern der LP-X-Nachweis als Cholestase-spezifisch angesehen werden kann. Dafür spricht auch, daß wir bei über $1 \mathrm{Jahr}$ alten Kindern positive LP-X-Teste immer klinisch-chemisch mit dem Vorliegen einer Cholestase korrelieren konnten.

Die Ursache des gehäuften Auftretens von LP-X bei Frühgeborenen und reifen Neugeborenen ist bisher noch unbekannt. Einige Möglichkeiten, die als Ursachen denkbar sind, konnten bereits ausgeschlossen werden:

Mit Sicherheit handelt es sich nicht um die fetale Form eines Lipoproteins, wie wir es von einigen Proteinen kennen, z. B. vom $\alpha_{1}$-Fetoprotein, vom Hämoglobin oder vom Fibrinogen. LP-X wurde von einer Ausnahme abgesehen nie am ersten Lebenstag nachgewiesen und trat häufig sogar erst auf, wenn die Kinder bereits einige Monate alt waren.

Mit diesem Befund ist auch eine ursprünglich zu den Angaben von Seidel et al. (3) bestehende Diskrepanz geklärt worden: Seidel et al. fanden im Nabelvenenblut kein LP-X und hielten daher den Test auch geeignet für das Neugeborenen-Alter. Da das Lipoprotein aber erst nach der Geburt auftritt, sind die Untersuchungen im Nabelvenenblut kein günștigẹ Vergleich für das Neugeborenen-Alter.

Da bei Erwachsenen mit angeborenem Mangel an LecithinCholesterol-Acyltransferase stets LP-X im Serum gefunden wird $(8,9)$, lag die Vermutung nahe, daß bei Neugeborenen die Aktivität des Enzyms vermindert sein könnte. Durch die Analyse des freien und veresterten Cholesterins (vĝl. Tab. 3) bei LP-X-negativen und -positiven Kindern konnte diese Ursache weitgehend ausgeschlossen werden, dénn ès wurden keine erhöhten
Konzentrationen an freiem Cholesterin gefunden. Da der Nachweis von LP-X in den vorliegenden Untersuchungen durch Polyanionen-Präzipitation erfolgte, wäre es auch möglich, daß es sich dabei um ein Lipoprotein handelt, das mit dem von Seidel et al. (1) beschriebenen Lipoprotein nicht identisch ist. Der LP-XNachweis wurde daher in einigen unserer Serumproben auch durch immunologische Präzipitation durchgeführt. Die bei Polyanionen-Präzipitation positiven Proben waren auch bei immunologischer Präzipitation positiv ${ }^{1}$. An der Identität der Lipoproteine besteht also kein Zweifel.

Bei den untersuchten Neugeborenen wurden klinischchemisch keinerlei Hinweise für eine Cholestase gefunden. Die Höhe der Gesamt-Bilirubin-Konzentration war ohne Einfluß auf das Vorkommen von LP-X und die sogenannten „Cholestase-anzeigenden“ Enzyme LeucinArylamidase und $\boldsymbol{\gamma}$-Glutamyltransferase waren bei den LP-X-positiven Kindem nicht erhöht.

So entsteht die Frage, ob das ,physiologische " Auftreten von LP-X bei Frühgeborenen und reifen Neugeborenen mit einer Cholestase .verbunden ist, die mit unseren üblichen Methoden nicht erfaßt werden kann, oder ob LP-X im Serum vorkommen kann, ohne daß eine Cholestase vorliegt.

Diese Frage ist bisher nicht zu beantworten und soll durch histologische Untersuchungen geklärt werden. Am einfachsten ist das gehäufte Auftreten von LP-X durch eine ,physiologische Unreife“ der Leberfunktion der Neugeborenen zu erklären. Es gibt zahlreiche Beispiele dafür, daß Enzymsysteme erst im Laufe des ersten Lebensjahres die Aktivitäten erreichen, die auch bei älteren Kindern und Erwachsenen gefunden werden. Da bei Neugeborenen durch die „physiologisch“ verminderte Gallensäuresynthese auch die Lipidabsorption herabgesetzt ist (10), ist auch ein abnormales Lipoprotein-Muster denkbar. Diese Annahme wäre vereinbar mit der Beobachtung, daß das abnormale Lipoprotein erst postnatal nach dem Beginn der Nahrungsaufnahme auftritt. Unverständlich ist allerdings, warum viele Kinder erst im Alter von 4-8 Wochen LP-X-positiv werden (vgl. Tab. 4 und 5). Die Vermutung, daß Kinder nach Austauschtransfusionen häufiger LP-X-positiv sind, muß in einer gezielten Untersuchung überprüt werden. Ebenso bedürfen die sich aus dem Vorkommen des abnormalen Lipoproteins bei Neugeborenen ergebenden biochemischen Probleme weiterer Analysen.

1) Wir danken Herrn Prof. Dr. D. Seidel, Heidelberg, für die Durchfuihrung der Analysen. 


\section{Literatur}

1. Seidel, D. \& Alaupovic, P. (1970), Deut. Med. Wochenschr.

7. Jendrassik, L. \& Gróf, P. (1938), Biochem. Z. 297, 81-89. 95, 1774-1780.

2. Seidel, D., Gretz, H. \& Ruppert, C. (1973), Clin. Chem. 19, 86-91.

3. Seidel, D., Schmitt, E. A. \& Alaupovic, P. (1970), Deut. Med. Wochenschr. 95, 1805-1809

4. Ritland, S., Blomhoff, J. P., Elgjo, K. \& Gjone, E. (1973), Scand. J. Gastroent. 8, 155-160.

5. Obcr, M. \& Witt, I. (1975), diese Z. 13, 256.

6. Lachmann, D., Bugajer-Gleitmann, Eder, G. \& Pollack, A. (1975), diese Z. 13, 257.

8. Torsvik, H., Berg, K., Magnani, H. N., McConathy, W. J., Alaupovic, P. \& Gjone, E. (1972), FEBS Letters 24, 165 bis 168.

9. Ritland, S. \& Gjone, E. (1975), Clin. Chim. Acta 59, 109 bis 119.

10. Watkins, J. B., Ingall, D., Szczepanik, P., Klein, P. D. \& Lester, R. (1973), New Engl. J. Med. 288, 431-438.

Prof. Dr. Irene Witt

Biochemisches Labor

Universitäts-Kinderklinik

D-7800 Freiburg i. Br.

Mathildenstr. 1 